DR. STEPHEN R ATKINSON (Orcid ID : 0000-0002-6910-7100)

PROF. RUDOLF E. STAUBER (Orcid ID : 0000-0002-3687-9331)

PROF. ALI CANBAY (Orcid ID : 0000-0001-6069-7899)

DR. LUCA VALENTI (Orcid ID : 0000-0001-8909-0345)

DR. JENS U. MARQUARDT (Orcid ID : 0000-0002-8314-2682)

DR. PIERRE DELTENRE (Orcid ID : 0000-0002-4253-6028)

Article type : Original

\title{
Genetic variation in HSD17B13 reduces the risk of developing cirrhosis and hepatocellular carcinoma in alcohol misusers
}

Short title: Alcoholic cirrhosis, HCC, risk reduction and HSD17B13

Felix Stickel ${ }^{1 *}$, Philipp Lutz $^{2^{*}}$, Stephan Buch ${ }^{3 *}$, Hans Dieter Nischalke ${ }^{2 *}$, Ines Silva ${ }^{4}$, Vanessa Rausch $^{4}$, Janett Fischer ${ }^{5}$, Karl Heinz Weiss ${ }^{6}$, Daniel Gotthardt ${ }^{6}$, Jonas Rosendahl7, Astrid Marot ${ }^{8}$, Mona Elamly ${ }^{8}$, Marcin Krawczyk ${ }^{9,29}$, Markus Casper ${ }^{9}$, Frank Lammert ${ }^{9}$, Thomas WM Buckley ${ }^{10}$, Andrew McQuillin ${ }^{10}$, Ulrich Spengler ${ }^{2}$, Florian Eyer ${ }^{11}$, Arndt Vogel ${ }^{12}$, Silke Marhenke ${ }^{12}$, Johann von Felden ${ }^{13}$, Henning Wege ${ }^{13}$, Rohini Sharma ${ }^{14}$, Stephen Atkinson ${ }^{14}$, Andre Franke ${ }^{15}$, Sophie Nehring ${ }^{3}$ Vincent Moser ${ }^{3}$, Clemens Schafmayer ${ }^{16}$, Laurent Spahr ${ }^{17}$, Carolin Lackner ${ }^{18}$, Rudolf E. Stauber ${ }^{19}$, Ali Canbay ${ }^{20}$, Alexander Link ${ }^{20}$, Luca Valenti ${ }^{21}$, Jane I. Grove ${ }^{22}$, Guruprasad P. Aithal22, Jens U. Marquardt ${ }^{23}$, Waleed Fateen ${ }^{22}$, Steffen Zopf ${ }^{24}$, Jean-Francois Dufour ${ }^{25}$, Jonel Trebicka ${ }^{26}$, Christian Datz $^{27}$, Pierre Deltenre ${ }^{8 \ddagger}$, Sebastian Mueller ${ }^{4 \ddagger}$, Thomas Berg ${ }^{5 \ddagger}$, Jochen Hampe ${ }^{3 \ddagger}$, Marsha Y Morgan ${ }^{28 \neq}$

*FS, PL, SB and HDN have contributed equally to the presented work and share premier authorship łPD, SM, TB, JH and MYM have contributed equally to the presented work and share senior authorship

${ }^{1}$ Department of Gastroenterology and Hepatology, University Hospital of Zurich, Switzerland

${ }^{2}$ Department of Internal Medicine I, University of Bonn, Bonn, Germany

This article has been accepted for publication and undergone full peer review but has not been through the copyediting, typesetting, pagination and proofreading process, which may lead to differences between this version and the Version of Record. Please cite this article as doi: 10.1002/HEP.30996

This article is protected by copyright. All rights reserved 
${ }^{3}$ Medical Department 1, University Hospital Dresden, TU Dresden, Germany

${ }^{4}$ Department of Internal Medicine and Center for Alcohol Research, Salem Medical Center University Hospital Heidelberg, Heidelberg, Germany

${ }^{5}$ Hepatology Section, Division of Gastroenterology and Rheumatology, University Hospital Leipzig, Leipzig, Germany

${ }^{6}$ Department of Internal Medicine IV, Medical University of Heidelberg, Germany

${ }^{7}$ Department of Gastroenterology, University Hospital Halle/Saale, Germany

${ }^{8}$ Division of Gastroenterology and Hepatology, Centre Hospitalier Universitaire Vaudois, University of Lausanne, Switzerland

${ }^{9}$ Department of Medicine II, Saarland University Medical Center, Homburg, Germany

${ }^{10}$ Molecular Psychiatry Laboratory, Division of Psychiatry, University College London, UK

${ }^{11}$ Department of Clinical Toxicology, Klinikum Rechts der Isar, Technical University of Munich, Germany

${ }^{12}$ Department of Gastroenterology, Hepatology and Endocrinology, Hannover Medical School, Germany

${ }^{13}$ First Department of Medicine, University Medical Center Hamburg-Eppendorf, Hamburg

${ }^{14}$ Department of Medicine, Division of Surgery and Cancer, Imperial College London, London, UK

${ }^{15}$ Institute of Clinical Molecular Biology, Kiel University, Kiel, Germany

${ }^{16}$ Department of Visceral and Thoracic Surgery, Kiel University, Kiel, Germany

${ }^{17}$ Departments of Gastroenterology and Hepatology, University Hospitals of Geneva and Faculty of Medicine, Geneva, Switzerland

${ }^{18}$ Institute of Pathology, Medical University of Graz, Austria

${ }^{19}$ Division of Gastroenterology and Hepatology, Department of Internal Medicine, Medical University of Graz, Austria

${ }^{20}$ Department of Gastroenterology, Hepatology and Infectious Diseases, Otto-von-Guericke University, Magdeburg, Germany

${ }^{21}$ Department of Pathophysiology and Transplantation, Università degli Studi di Milano, and Translational

Medicine - Department of Transfusion Medicine and Hematology, Fondazione IRCCS Ca'Granda Ospedale Maggiore Policlinico, Milan, Italy

${ }^{22}$ NIHR Nottingham Biomedical Research Centre, Nottingham University Hospitals NHS Trust and the University of Nottingham, Nottingham, UK; Nottingham Digestive Diseases Centre, University of Nottingham, Nottingham, UK

${ }^{23}$ Department of Medicine I, Johannes Gutenberg-Universität Mainz, Mainz, Germany

${ }^{24}$ Medical Department 1, University of Erlangen-Nuremberg, Germany

${ }^{25}$ University Clinic for Visceral Surgery and Medicine, Inselspital, University of Berne, Berne, Switzerland

This article is protected by copyright. All rights reserved 
${ }^{26}$ Department of Internal Medicine I, Goethe University Frankfurt, Frankfurt, Germany

${ }^{27}$ Department of Internal Medicine, Hospital Oberndorf, Teaching Hospital of the Paracelsus Private Medical University of Salzburg, Oberndorf, Austria

${ }^{28}$ UCL Institute for Liver \& Digestive Health, Division of Medicine, Royal Free Campus, University College London, United Kingdom

${ }^{29}$ Laboratory of Metabolic Liver Diseases, Department of General, Transplant and Liver Surgery, Centre for Preclinical Research, Medical University of Warsaw, Warsaw, Poland

\section{Correspondence}

Felix Stickel, MD

Department of Gastroenterology and Hepatology, University Hospital Zürich, Rämistrasse 100, CH-8091 Zürich, Switzerland

Phone +41-31-338 7817

Fax +41-31-335 3519

Felix.stickel@uzh.ch

Key words: adiponutrin; candidate genes; fibrosis; genetic risk association; genetic susceptibility; host genetics; lipotoxicity.

\section{Word count: 6,012}

Tables: 3 (+ 8 Supplementary Tables)

Figures: 3 (+ 2 Supplementary Figures $1 \mathrm{~A}-\mathrm{C}, 2 \mathrm{~A}-\mathrm{C}$ )

\section{Conflicting Interests}

None of the authors has any conflict of interest in relation to the contents of this manuscript.

\section{Funding}

This work was supported by grants from the Swiss National Funds (SNF no. 310030_169196) and the Swiss Foundation for Alcohol Research (SSA) to FS, and the Liver Systems Medicine (LiSyM) Network funded by the German Federal Ministry for Education and Research (BmBF) to JH. HDN and US were supported by a grant from the Deutsche Krebshilfe (70112169). 


\section{Author contributions}

Felix Stickel

Philipp Lutz

Stephan Buch

Hans Dieter Nischalke

Ines Silva

Vanessa Rausch

Janett Fischer

Karl Heinz Weiss

Daniel Gotthardt

Jonas Rosendahl

Astrid Marot

Mona Elamly

Marcin Krawczyk

Markus Casper

Frank Lammert

Thomas Buckley conceived the study, raised the pivotal funding, contributed biological material from cases and controls, analyzed the data, wrote the manuscript, acted as the corresponding author and approved the final draft submitted

contributed biological material from cases and controls, analyzed the data, critically revised the manuscript and approved the final draft submitted analyzed the primary data, performed the biostatistics, critically revised the manuscript and approved the final draft submitted

contributed biological material from cases and controls, analyzed the data, critically revised the manuscript and approved the final draft submitted

contributed biological material from phenotyped cases and controls, critically revised the manuscript and approved the final draft submitted

contributed biological material from phenotyped cases and controls, critically revised the manuscript and approved the final draft submitted

contributed biological material from phenotyped cases and controls, critically revised the manuscript and approved the final draft submitted

contributed biological material from phenotyped cases and controls, critically revised the manuscript and approved the final draft submitted

contributed biological material from phenotyped cases and controls, critically revised the manuscript and approved the final draft submitted

contributed biological material from phenotyped cases and controls, critically revised the manuscript and approved the final draft submitted

contributed biological material from phenotyped cases and controls, critically revised the manuscript and approved the final draft submitted

contributed biological material from phenotyped cases and controls, critically revised the manuscript and approved the final draft submitted

contributed biological material from phenotyped cases and controls, critically revised the manuscript and approved the final draft submitted

contributed biological material from phenotyped cases and controls, critically revised the manuscript and approved the final draft submitted

contributed biological material from phenotyped cases and controls, critically revised the manuscript and approved the final draft submitted genotypes the healthy control samples collected in the United Kingdom, critically revised the manuscript and approved the final draft submitted 
Andrew McQuillin

Ulrich Spengler

Florian Eyer

Arndt Vogel

Silke Marhenke

Johann von Felden

Henning Wege

Rohini Sharma

Stephen Atkinson

Andre Franke

Sophie Nehring

Vincent Moser

Clemens Schafmayer

Laurent Spahr

Carolin Lackner

Rudolf E. Stauber

Ali Canbay contributed biological material from healthy controls, reviewed the biostatistics analysis, critically revised the manuscript and approved the final draft submitted contributed biological material from phenotyped cases and controls, critically revised the manuscript and approved the final draft submitted

contributed biological material from phenotyped cases and controls, critically revised the manuscript and approved the final draft submitted

contributed biological material from phenotyped cases and controls, critically revised the manuscript and approved the final draft submitted

contributed biological material from phenotyped cases and controls, critically revised the manuscript and approved the final draft submitted

contributed biological material from phenotyped cases and controls, critically revised the manuscript and approved the final draft submitted

contributed biological material from phenotyped cases and controls, critically revised the manuscript and approved the final draft submitted

contributed biological material from phenotyped cases and controls, critically revised the manuscript and approved the final draft submitted

contributed biological material from phenotyped cases and controls, critically revised the manuscript and approved the final draft submitted

contributed biological material from phenotyped cases and controls, critically revised the manuscript and approved the final draft submitted

contributed biological material from phenotyped cases and controls, critically revised the manuscript and approved the final draft submitted

contributed biological material from phenotyped cases and controls, critically revised the manuscript and approved the final draft submitted

contributed biological material from phenotyped cases and controls, critically revised the manuscript and approved the final draft submitted

contributed biological material from phenotyped cases and controls, critically revised the manuscript and approved the final draft submitted

contributed biological material from phenotyped cases and controls, critically revised the manuscript and approved the final draft submitted

contributed biological material from phenotyped cases and controls, critically revised the manuscript and approved the final draft submitted

contributed biological material from phenotyped cases and controls, critically revised the manuscript and approved the final draft submitted 
Alexander Link

Luca Valenti

Jane I. Grove

Guruprasad P. Aithal

Jens U. Marquardt

Waleed Fateen

Steffen Zopf

Jean-Francois Dufour

Jonel Trebicka

Christian Datz

Pierre Deltenre

Sebastian Mueller

Thomas Berg

Jochen Hampe

Marsha Y. Morgan contributed biological material from phenotyped cases and controls, critically revised the manuscript and approved the final draft submitted contributed biological material from phenotyped cases and controls, critically revised the manuscript and approved the final draft submitted contributed biological material from phenotyped cases and controls, critically revised the manuscript and approved the final draft submitted contributed biological material from phenotyped cases and controls, critically revised the manuscript and approved the final draft submitted contributed biological material from phenotyped cases and controls, critically revised the manuscript and approved the final draft submitted contributed biological material from phenotyped cases and controls, critically revised the manuscript and approved the final draft submitted contributed biological material from phenotyped cases and controls, critically revised the manuscript and approved the final draft submitted contributed biological material from phenotyped cases and controls, critically revised the manuscript and approved the final draft submitted contributed biological material from phenotyped cases and controls, critically revised the manuscript and approved the final draft submitted contributed biological material from phenotyped cases and controls, critically revised the manuscript and approved the final draft submitted contributed biological material from phenotyped cases and controls, critically revised the manuscript and approved the final draft submitted contributed a large amount of biological material from phenotyped cases and controls, critically revised the manuscript and approved the final draft submitted contributed a large amount of biological material from phenotyped cases and controls, critically revised the manuscript and approved the final draft submitted conceived the study, provided the genotyping infrastructure, critically revised the manuscript and approved the final draft submitted conceived the study, contributed biological material from phenotyped cases and controls, re-analyzed the data, co-wrote the manuscript, critically revised the manuscript and approved the final draft submitted 


\section{ABSTRACT}

Background \& Aims: Carriage of rs738409:G in patatin-like phospholipase domain-containing 3 (PNPLA3) is associated with an increased risk for developing alcohol-related cirrhosis and hepatocellular carcinoma (HCC). Recently, rs72613567:TA in hydroxysteroid 17-beta dehydrogenase 13 (HSD17B13) was shown to be associated with a reduced risk for developing alcohol-related liver disease and to attenuate the risk associated with PNPLA3 rs738409:G. This study explores the risk-associations between these two genetic variants and the development of alcohol-related cirrhosis and HCC.

Approach and Results: Variants in HSD17B13 and PNPLA3 were genotyped in 6,171 participants, including: 1,031 with alcohol-related cirrhosis and HCC; 1,653 with alcohol-related cirrhosis without HCC; 2,588 alcohol misusers with no liver disease; and 899 healthy controls. Genetic associations with the risks for alcohol-related cirrhosis and HCC were determined using logistic regression analysis. Carriage of HSD17B13 rs72613567:TA was associated with a lower risk for both cirrhosis (OR 0.79 [95\% Cl 0.72-0.88], $\left.\mathrm{p}=8.13 \times 10^{-6}\right)$ and $\mathrm{HCC}\left(\mathrm{OR} 0.77\right.$ [95\% Cl 0.68-0.89], $\left.\mathrm{p}=2.27 \times 10^{-4}\right)$, while carriage of PNPLA3 rs738409:G was associated with an increased risk for developing cirrhosis (OR 1.70 [95\% $\mathrm{Cl} 1.54-1.88$ ], $\mathrm{p}=1.52 \times 10^{-26}$ ) and HCC (OR 1.77 [95\% Cl 1.58-1.98], $p=2.31 \times 10^{-23}$ ). These associations remained significant after adjusting for age, sex, body mass index, type II diabetes mellitus and country. Carriage of HSD17B13 rs72613567:TA attenuated the risk for developing cirrhosis associated with PNPLA3 rs738409:G in both men and women but the protective effect against the subsequent development of HCC was only observed in men $\left(p=1.72 \times 10^{-4} ; O_{\text {allelic }}, 0.75 ; 95 \% \mathrm{Cl}, 0.64-0.87\right)$.

Conclusions: Carriage of variants in PNPLA3 and HSD17B13 differentially affect the risk for developing advanced alcohol-related liver disease. A genotypic/phenotypic risk score might facilitate earlier diagnosis of HCC in this population.

Word count: 273

\section{INTRODUCTION}

Alcohol-related liver disease has a global prevalence of $12 \%$ and accounts for half of all cirrhosisassociated deaths in Europe and the United States. ${ }^{1-3}$ Alcohol-related cirrhosis is now the second most common indication for liver transplantation, accounting for approximately $40 \%$ of all primary liver transplants in Europe and approximately 25\% in the United States. ${ }^{4}$

This article is protected by copyright. All rights reserved 
Chronic alcohol misuse is associated with the development of a broad spectrum of liver injury. Hepatic steatosis develops in most heavy alcohol users, but more substantial liver injury only develops with persistent alcohol misuse over time; inflammation and progressive fibrosis will develop in 10 to $35 \%$ of individuals, whereas cirrhosis is observed in only 10 to $15 \% .^{5-7}$ Between

5 to $15 \%$ of people with alcohol-related cirrhosis are at risk for developing hepatocellular carcinoma ( $\mathrm{HCC}$ ); the annual incidence is 2.5 to $3.0 \%$, with a 5 -year cumulative risk of around $8 \% .^{8-11}$ The global incidence of HCC is increasing; it is now the fifth most frequent cancer and third most frequent cause of cancer-related mortality world-wide; one third of cases develop on a background of alcohol-related cirrhosis. ${ }^{12}$

The susceptibility to develop significant alcohol-related liver disease is determined by the interplay of a number of risk factors, including sex, ethnicity, the amount/pattern of alcohol drinking, coffee consumption, cigarette smoking, co-morbidies such as obesity, type 2 diabetes and hepatitis $C$ infection and a number of host genetic factors. ${ }^{13}$ Carriage of the common missense variant rs738409:G in patatin-like phospholipase domain-containing 3 (PNPLA3) is the most robustly validated risk locus for the development of alcohol-related cirrhosis and HCC, ${ }^{14-19}$ accounting for $26.6 \%^{14}$ and $43.5 \%^{18}$ of the variance, respectively. Two further gene variants, rs58542926 in transmembrane 6 superfamily member 2 (TM6SF2) and rs641738 in membrane bound O-acetyltransferase domain containing 7 (MBOAT7), are additional risk factors, albeit with much lower effect sizes. ${ }^{19}$

Recently, Abul-Husn and colleagues ${ }^{20}$ identified a splice variant rs72613567 in hydroxysteroid 17beta dehydrogenase 13 (HSD17B13), which appeared to protect against the development of chronic liver injury in people of European ancestry. HSD17B13 encodes a hepatic lipid droplet protein; the insertion of an adenine adjacent to the donor splice site on exon 6 (rs72613567:TA allele) results in an unstable and truncated protein with reduced enzymatic activity, suggesting that it is a loss-of-function variant. ${ }^{20}$ Abul-Husn et al. ${ }^{20}$ found that carriage of HSD17B13 rs72613567:TA was associated with reduced serum aminotransferase activities, a reduced risk for developing alcohol-related and non-alcoholic fatty liver disease (NAFLD), and, more specifically, for developing both alcohol-related and NAFLD-related cirrhosis. ${ }^{20}$ The association with alcohol-related cirrhosis was the most compelling but the total number of cases was very 
small. This group also showed that carriage of this variant attenuated the risk of developing progressive liver injury derived from rs738409:G in PNPLA3. ${ }^{20}$

Yang et al. ${ }^{21}$ recently confirmed the association between rs72613567:TA in HSD17B13 and a reduction in the risk for developing alcohol-, NAFLD- and hepatitis C-related liver disease, and more specifically the risk of developing cirrhosis. They also found that carriage of this variant protected against the development of HCC, but only in patients with a background of alcoholrelated liver disease. The sample size was small and those with HCC were more frequently male and older than their counterparts without HCC. Thus, in order to validate these findings much larger cohorts are needed to allow adjustments for potential confounders. ${ }^{21}$ Interactions between variants in HSD17B13 and PNPLA3 were not addressed in this study.

The aims of the present study were to determine whether: (i) carriage of rs72613567:TA in HSD17B13 protects against the development of alcohol-related cirrhosis and alcohol-related HCC; and (ii) to explore possible risk interactions between rs72613567:TA in HSD17B13 and rs738409:G in PNPLA3. 


\section{METHODS}

\section{Study cohorts}

The study population comprised of 6,171 participants drawn from one of four study cohorts:

1. Alcohol-related cirrhosis and HCC (HCC) $(n=1,031)$

2. Alcohol-related cirrhosis without HCC (CIRR) $(n=1,653)$

3. Alcohol misusers with no evident liver disease (ALC) $(n=2,588)$

4. Healthy controls with no history of alcohol misuse or liver disease $(n=899)$

Participants in cohorts 1-3 were recruited from Hepatology units and Addiction centres across Europe and were of self-reported Swiss/German/Austrian/Italian/British ancestry. Ninety of the UK samples with cirrhosis and HCC were obtained from the Nottingham Digestive Diseases Centre Biomedical Research Unit Research Tissue Bank (Rec Ref: 14/WA/1234). Participants in cohort 4 were recruited from the United Kingdom.

The diagnosis of alcohol-related cirrhosis was established, as described, in detail, previously; ${ }^{19}$ briefly, the diagnosis was based on a history of prolonged, sustained alcohol intake of a minimum of $40 \mathrm{~g} /$ day in women and $60 \mathrm{~g} /$ day in men together with histological examination of liver tissue; or compatible historical, clinical, laboratory, radiological and endoscopic features of advanced chronic liver disease. Patients were excluded if they had any other potential cause of liver injury, specifically if they were positive for hepatitis B surface antigen (HBsAg), anti-hepatitis C immunoglobulin G (anti-HCV IgG), anti-nuclear antibodies (ANA) (titre $>1: 80$ ) or antimitochondrial antibodies (titre $>1: 40$ ). Patients with elevated serum ferritin levels and a transferrin saturation of $>50 \%$, a serum caeruloplasmin of $<20 \mathrm{mg} / \mathrm{dl}(0.2 \mathrm{~g} / \mathrm{dl})$ or a serum alpha-1 antitrypsin of $<70 \mathrm{mg} / \mathrm{dL}(13 \mu \mathrm{mol} / \mathrm{L})$ were further investigated and excluded, as appropriate.

The diagnosis of HCC was based on histological examination of tumour tissue or evidence on imaging, preferably using two modalities, of lesions that were hypervascular in the arterial phase with washout in the portal venous or delayed phases. ${ }^{22}$ The severity of the underlying cirrhosis was assessed using Pugh's modification of the Child's grading system. ${ }^{23}$

The patients with alcohol misuse but no evidence of significant liver injury were recruited as described, in detail, previously; ${ }^{19}$ in brief, they had a background of alcohol consumption of at 
least $60 \mathrm{~g} / \mathrm{d}$ for women and $80 \mathrm{~g} / \mathrm{d}$ for men for $\geq 10$ years with or without features of alcohol dependence. $^{24}$ None had historical, clinical or laboratory evidence of liver disease and its absence was confirmed either by a liver stiffness measurement (Fibroscan, Echosens, Paris) of below $6 \mathrm{kPa}(\mathrm{IQR}<20 \%)$ or by the absence of histological liver damage.

Healthy controls were recruited from London branches of the National Health Service blood transfusion service, from General Practitioners' surgeries, from amongst university students, and from the general public. None currently drank alcohol above a weekly maximum of $112 \mathrm{~g}$ for women and $168 \mathrm{~g}$ for men, nor had they done so at any time in the past. None had a history or clinical evidence of liver disease

\section{DNA preparation and genotyping}

Genomic DNA was extracted from venous blood samples and quantified using standard procedures. ${ }^{19}$ Genotyping of PNPLA3 rs738409 (Assay ID: C_7241_10) and HSD17B13 rs72613567 (primer and probe sets manufactured through custom TaqMan ${ }^{\circledR}$ Assay design) was performed using TaqMan ${ }^{\circledR}$ SNP Genotyping Assays and Chemistries (Applied Biosystems, Waltham, MA, USA) on an automated platform with TECAN Freedom EVO and 384well TEMO liquid handling robots (TECAN, Männedorf, Switzerland) as described previously. ${ }^{18,19}$

All process data were logged and administered with a database-driven LIMS. Reactions were completed and read in a 7900 HT TaqMan sequence detector system (Applied Biosystems, Waltham, MA, USA). The amplification reaction was carried out with the TaqMan universal master mix at cycling conditions of 1 cycle for 10 minutes at $95^{\circ} \mathrm{C}$, followed by 45 cycles for 15 seconds at $95^{\circ} \mathrm{C}$, and 1 minute at $60^{\circ} \mathrm{C}$.

\section{Statistical analysis}

Logistic regression and SNP*SNP interaction analyses were performed using SPSS v.25.0 (IBM Corp., Armonk, NY, USA). A three-way case control design was adopted: HCC vs. CIRR; CIRR vs. ALC; HCC vs. ALC. Genotypic and allelic tests of association were assessed using two logistic regression models:

Model A: univariate logistic regression; 
Model B: adjustments for age, sex, body mass index (BMI), type 2 diabetes mellitus (T2DM) and country.

Results, expressed as odds ratios (OR) with their $95 \%$ confidence intervals $(\mathrm{Cl})$, were derived from beta coefficients and their standard deviations. Nominal two-sided asymptotic $p$-values are reported for all tests.

The interactions between HSD17B13 and PNPLA3 were examined by logistic regression for the univariate additive and genotypic regression models including both main SNP effects and the SNP*SNP interaction term.

Fixed-effect model meta-analysis using the inverse variance-weighted method to summarize effect sizes and forest plots were performed using the R package 'metafor' v.2.0-0.

Sex-specific post-hoc analyses for the risk associations with HSD17B13 and PNPLA3 were performed. The Mantel-Haenszel test for trend was applied for testing a linear trend in observed genotype proportions from contingency tables for $(A L C<C I R R<H C C)$, by sex.

The population-attributable fraction (PAF) provides an epidemiological estimate of the proportion of the disorder that is attributable to a given risk factor. Thus, in this instance it is an estimate of how much lower the frequency of HCC would be in patients with alcohol-related cirrhosis, if the risk genotype(s) were eliminated from the population.

The PAF was estimated for heterozygous and homozygous carriage using the formula: ${ }^{25}$

$$
(x-1) / x
$$

where $x=(1-p)^{2}+2 p(1-p) O R 1+p^{2}$ OR2 (19); $p$ is the allele frequency in the CIRR or ALC cohorts, and OR1 and OR2 are the ORs associated with hetero- and homozygosity.

Combined PAF estimates were calculated as PAF=1-(1-PAF1) (1-PAF2) (1-PAFn) based on the individual PAFs for each associated SNP, assuming no multiplicative interaction between them.

\section{Ethics}

The study protocol was approved by the ethics committees of the participating institutions; all included subjects provided written informed consent prior to inclusion into the study. 


\section{RESULTS}

The patient cohorts were predominantly male and middle aged. The patients with cirrhosis were generally older than those misusing alcohol and more likely to be overweight and diabetic. The patients with cirrhosis and HCC were generally older than the patients with cirrhosis without malignant transformation and were proportionately more likely again to be male, overweight, and diabetic (Table 1). Laboratory variables showed the expected gradients (Supplementary Table 1).

Genotyping was completed for both SNPs with call rates greater than 95\% (Supplementary Table 2). All markers followed Hardy-Weinberg equilibrium (cutoff HWE $P>0.05$ ). ${ }^{26}$ In the healthy controls the minor allele frequencies (MAFs) for both HSD17B13 rs72613567:TA (0.276) and PNPLA3 rs738409 G (0.224) were comparable to those in public databases ${ }^{27}$ and previous publications ${ }^{18-20}$ and did not differ significantly from the MAFs in the ALC group (Supplementary Table 2).

A progressive reduction in MAFs for HSD17B13 rs72613567:TA was observed between the three groups: ALC (26.4\%); CIRR (22.0\%) and HCC (17.7\%) $\left(P_{\text {trend }}=1.09 \times 10^{-15}\right)$ (Supplementary Table 2). This contrasted with the expected stepwise increase in the MAFs for PNPLA3 rs738409:G in the same three groups viz. ALC (23.9\%) CIRR (35.4\%), HCC (49.8\%) (Supplementary Table 2).

In the univariate model, allelic and genotypic associations for HSD17B13 rs72613567:TA were highly significant for the comparisons HCC vs. CIRR $\left(P_{\text {allelic }}=2.27 \times 10^{-4}, P_{\text {genotypic }}=1.05 \times 10^{-3}\right), \mathrm{CIRR}$ vs. $\mathrm{ALC}\left(\mathrm{P}_{\text {allelic }}=8.13 \times 10^{-6}, \mathrm{P}_{\text {genotypic }}=1.54 \times 10^{-6}\right)$, and HCC vs ALC $\left(\mathrm{P}_{\text {allelic }}=1.69 \times 10^{-14}, \mathrm{P}_{\text {genotypic }}=\right.$ $3.22 \times 10^{-14}$ ) (Table 2). The protective effect associated with carriage of HSD17B13 rs72613567:TA remained significant in the CIRR and HCC cohorts after correction for sex, age, BMI, T2DM and country (Table 2). The protective effect for HCC was greater with homozygous than heterozygous carriage of the HSD17B13 rs72613567:TA allele (Figure 1A+B).

Allelic and genotypic associations for PNPLA3 rs738409:G allele were also significantly associated in the CIRR $\left(\mathrm{OR}_{\text {allelic }} 1.70\right.$ [1.54-1.88], $\left.\mathrm{P}_{\text {allelic }}=1.52 \times 10^{-26}\right)$ and $\mathrm{HCC}\left(\mathrm{OR}_{\text {allelic }} 1.77\right.$ [1.58-1.98], $\mathrm{P}_{\text {allelic }}=$ $2.31 \times 10^{-23}$ ) cohorts (Table 3). These associations were robust to corrections for sex, age, BMI, DMT2 and country.

This article is protected by copyright. All rights reserved 
Since patients were recruited from across Europe a meta-analysis of the HSD17B13 and PNPLA3 loci for association with cirrhosis and HCC was performed, by country (Supplementary Tables 3+4). Associations were calculated consensually, but were not necessarily significant in each single European cohort. However, fixed effect meta-analysis on aggregate confirmed both the protective and risk-enhancing effects of HSD17B13 and PNPLA3 loci, respectively, in the total cohort. There was no evidence of heterogeneity (Supplementary Figures $1 \mathrm{~A}-\mathrm{C}$ and $2 \mathrm{~A}-\mathrm{C}$ ).

The PAF for PNPLA3 rs738409 for CIRR was $27.2 \%$ (20.7\% to $33.6 \%)$ and for HSD17B13 rs72613567 $-14.7 \%$ (-21.2\% to -7.9\%). The combined PAF for CIRR was $16.5 \%$. The PAF\% for PNPLA3 rs738409 in HCC was 38.7\% (29.3\% to 47.2\%) and for HSD17B13 rs72613567 -10.0\% ($16.7 \%$ to $-2.7 \%)$. The combined PAF for HCC was $32.6 \%$.

The potential modifying effect of HSD17B13 rs72613567 on PNPLA3 rs738409 was explored by calculating the proportion of the HCC, CIRR and ALC cohorts with different 'gene signatures' combining either PNPLA3 rs739409:G (high risk variant) with wild type HSD17B13 rs72613567:T (lack of protection), or the PNPLA3 rs739409 C (low risk variant) with HSD17B13 rs72613567:TA (protection). A preponderance of patients in the CIRR and HCC cohorts were homozygous for PNPLA3 rs739409 G and for HSD17B13 rs72613567:T which is in line with the estimated risk contributions of each variant (Figure $2 A \& B$ ).

Possible SNP*SNP interactions between HSD17B13 rs72613567 and PNPLA3 rs739409 were explored by testing interaction terms in the logistic regression models for the risk for developing $\mathrm{CIRR}$ and the further risk for developing HCC. The genotypic interactions for the risk association with CIRR was not significant in the total study population $(p=0.598)$ (Supplementary Table 5) nor in the men only cohort $(p=0.689)$ (Supplementary Table 6). None of the HSD17B13 by PNPLA3 genotype interaction pair combinations was significant. The interactions in the additive CIRR and HCC risk models were not significant either. Thus, there does not appear to be an HSD17B13 rs72613567:TA × PNPLA3 rs739409:G interaction.

In a sex-specific post hoc analysis of the HSD17B13 locus, an association with HCC disease risk was observed in men $\left(P=1.72 \times 10^{-4} ; \mathrm{OR}_{\text {allelic, }} 0.75 ; 95 \% \mathrm{Cl}, 0.64-0.87\right)$ but not in women $(\mathrm{p}=$

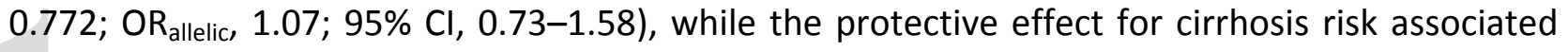
with carriage of HSD17B13 rs72613567:TA was observed in both sexes (Supplementary Table 7; 
Figures 3A-C). The association between carriage of PNPLA3 rs739409:G and cirrhosis and HCC disease risk showed no sex differential (Supplementary Table 8). 


\section{DISCUSSION}

The genetic contribution to the development of significant alcohol-related liver injury is gradually being unraveled. Three genetic loci have been identified which are associated with an increased risk for developing cirrhosis at genome-wide significance, namely PNPLA3, TM6SF2, MBOAT7, ${ }^{19}$ and at least two variants, PNPLA3 rs738409. and TM6SF2 rs58542926, are associated with an increased risk for developing HCC on this background. ${ }^{15,16,18}$

There is little published evidence for genetic variants conferring protection against the development of significant alcohol-related liver injury. Indirect protection is afforded in individuals, primarily of East Asian ancestry, who carry rs1229984 in alcohol dehydrogenase IB $(A D H 1 B)^{28}$ and/or rs671 in aldehyde dehydrogenase $2(A L D H 2)^{29}$ and, as a consequence, develop an abreaction to alcohol and so tend to avoid it.

Recently, however, Abul-Husn and colleagues $^{20}$ identified a splice variant, rs72613567, in HSD17B13, which appeared to protect against the development of chronic liver injury in people of European ancestry. Specifically, this SNP appeared to confer protection against the development of alcohol-related and NAFLD-related cirrhosis. ${ }^{20}$ The association with alcoholrelated cirrhosis was more compelling even though the total number of cases was very small, amounting to only 124 in their discovery cohort and 215 in their validation cohort.

Yang and coworkers, ${ }^{21}$ in a recent French/Belgian collaboration confirmed that rs72613567 in HSD17B13 confers protection against the development of alcohol-, NAFLD- and HCV-related liver disease. They further showed that this SNP confers protection against the development of HCC but only when arising on a background of alcohol-related liver disease. The data were adjusted for age, sex and the degree of hepatic fibrosis but not for other important confounders such as BMI, diabetes and PNPLA3 genotype. Only half the patients with alcohol-related HCC, in this study, had cirrhosis. Thus the numbers of patients with alcohol-related cirrhosis $(n=1243)$ and alcohol-related HCC $(n=217)$ were relatively small and those with HCC were more frequently male and older than those with cirrhosis per se. Adjustments were not made for these possible confounders leading the authors to conclude that larger cohorts were needed to validate their findings. 
The cohorts included in the present study were large, comprising of 1,031 patients of European ancestry with alcohol-related cirrhosis and HCC, and 1,653 with alcohol-related cirrhosis without HCC. In addition, 2,588 alcohol misusers, with no evidence of liver disease, were included to exclude the possibility that any observed genetic effect might relate to the susceptibility to develop problematic drinking.

The results of the present study robustly establish that carriage of HSD17B13:rs72613567:TA protects against the development of alcohol-related cirrhosis and its subsequent evolution to HCC. The results also confirm again that carriage of PNPLA3 rs738409 is associated with a significantly increased risk of developing alcohol-related cirrhosis and HCC. However, the magnitude of these two effects is of a different order. Thus, while carriage of the HSD17B13:rs72613567:TA allele reduces the PAF for cirrhosis by $14.7 \%$ and for HCC by $10 \%$, carriage of the PNPLA3 rs738409:G allele increases the risks by $27.2 \%$ and $38.7 \%$ respectively. Thus, the combined PAF for cirrhosis was $16.5 \%$ and for HCC $32.5 \%$.

Thus, the risk of carriage of PNPLA3 rs738409:G appears to be attenuated, to a degree, by cocarriage of HSD17B13:rs72613567:TA. Abul-Husn and colleagues ${ }^{20}$ explored the possibility of interactions between these two SNPs and observed nominally significant interactions in association analyses with serum transaminase activities, primarily in the obese, but not with chronic liver disease. They also showed, using RNA sequencing-based expression analysis, that HSD17B13 rs72613567:TA was associated with decreased PNPLA3 messenger RNA expression in an allele dose-dependent manner. However, exploration of SNP*SNP interaction, in the present study, showed no evidence of a significant interaction. Yang and coworkers ${ }^{21}$ did not report data on possible SNP*SNP interaction so no further comparison can be made.

Male sex is a significant risk factor for the development of HCC in people with alcohol-related cirrhosis. $^{12,13}$ The reasons for this are unknown. Men are more likely to drink at harmful levels and hence more likely to develop alcohol-related cirrhosis but they are still proportionately over represented amongst those developing HCC. Malignant transformation is more likely to occur in individuals with alcohol-related cirrhosis who stop drinking; ${ }^{30}$ hence the suggestion that the difference in sex-specific HCC rates may reflect lower abstinence rates amongst women. The possibility of sex-genetic variant interactions should also be considered. The comparative 
survival advantage in women with alcohol-related cirrhosis is well-documented, ${ }^{31-33}$ and it has recently been shown that this may relate, at least in part, to a sex-variant interaction with rs738409:G in PNPLA3. 34,35

The possibility of sex-variant interactions was also explored in the present study. The progressive increase in the risk of developing alcohol-related cirrhosis and HCC associated with carriage of PNPLA3 rs738409:G HCC was observed in both men and women. Likewise, the protective effect for cirrhosis risk associated with carriage of HSD17B13 rs72613567:TA was observed in both sexes. However, the protective association of HSD17B13 rs72613567:TA and HCC was only found in men. There was no significant sex difference in the MAFs for HSD17B13 rs72613567:TA in the alcohol misusers $(26.3 \%$ vs. $26.6 \%)$ or in the patients with alcohol-related cirrhosis $(22.4 \%$ vs. 20.5\%). However, whereas the frequency further reduced in men with HCC (17.8\%) the trend partially reversed in women (21.7\%). These findings are counterintuitive and are without explanation. The number of women with HCC was relatively small so this finding needs further exploration in a larger cohort.

Neither of the previous studies explored sex-differences in the effects of HSD17B13 rs72613567:TA. ${ }^{20,21}$ However, Ferenci and colleagues, ${ }^{36}$ found sex-related phenotypic variation associated with carriage of HSD17B13 rs72613567:TA in patients with Wilson's disease. Thus, none of the in men who developed fulminant Wilson's disease carried this HAS17B13 variant in contrast to $13.6 \%$ in their female counterparts. Clearly, sex-gene variant interaction should be considered in any further genetic studies in the field of liver disease.

HSD17B13 belongs to the family of pluripotent 17-hydroxysteroid dehydrogenase enzymes whose members convert 17-keto- and 17-hydroxysteroids, regulate the biological activity of sex hormones, participate in fatty acid and cholesterol metabolism, and contribute to bile acid synthesis. ${ }^{37}$ The function of HSD17B13 is incompletely understood, but it is located on the surface of lipid droplets and is mainly expressed in the liver. Su and colleagues ${ }^{38}$ have demonstrated that transcriptional regulation of HSD17B13 expression is likely to be liver $\mathrm{X}$ receptor (LXR)-mediated via a sterol-regulatory-element binding protein 1c (SREBP-1c)dependent mechanism; they also found evidence for a SREBP-1c response element (SRE) in the promoter region of the HSD17B13 gene located on chromosome 4q22.1. HSD17B13 expression 
is increased in patients with NAFLD 39,40 and in murine models of fatty liver disease, ${ }^{41}$ and its overexpression in Huh-7 and HepG2 hepatoma cell lines results in accumulation of HSD17B13 on the surface of lipid droplets. ${ }^{40,42}$

$\mathrm{Ma}$ and coworkers ${ }^{43}$ have recently shown that HSD17B13 is a hepatic retinol dehydrogenase. Retinal, retinoic acid and retinol binding protein have been implicated in the pathogenesis of steatosis, fibrosis, adipogenesis, and insulin resistance. ${ }^{44}$ Thus, HSD17B13 may be involved in the complex nuclear receptor interaction in NAFLD via activation of the retinoic acid receptor. ${ }^{45}$ Of greater importance, in the context of the present study, is the possibility that HSD17B13, functioning as a retinol dehydrogenase, may contribute to the depletion of hepatic retinoic acid observed in individuals chronically misusing alcohol. Retinoic acid depletion results in a functional down regulation of liver retinoic acid receptors and a marked increase in the expression of the activator protein-1 (c-jun and c-fos) transcriptional complex which is associated with hepatic cell hyperproliferation, a decrease in apoptosis and stimulated hepatic carcinogenesis. ${ }^{46,47}$ The retinol dehydrogenase activity of the rs72613567:TA variant is likely reduced or absent and this may explain, at least in part, its protective effect against HCC in the context of alcohol-related cirrhosis. Accordingly, repletion of hepatic retinoic acid concentrations experimentally or in patients with HCC may have a therapeutic role. ${ }^{48}$

There are some contrary findings in relation to the protective effect of a reduction in HSD17B13 activity. Chen and colleagues, ${ }^{49}$ for example, reported that mRNA expression of HSD17B13 was down regulated in Asians with hepatitis B (HBV)-related HCC, though not in HBV-related cirrhosis, and showed that low HSD17B13 expression in peritumour tissue was independently associated with a reduction in recurrence-free survival. They also showed that overexpression of HSD17B13 in Huh-7 cell and SK-HEP-1 cell lines results in delays in cell cycle progression. Their overall conclusion, based on these findings, was that increased HSD17B13 expression might inhibit the development and progression of HBV-related HCC. This contrast with the findings in the present study and those of others, ${ }^{20,21}$ that in Europeans, decreased expression of HSD17B13 protects against the development of both alcohol-related cirrhosis and its evolution to HCC. This apparent contradiction may attest to differences in the mechanisms of HCC development in viral- 
and alcohol-related liver disease. Clearly further research on the functional role of HSD17B13 in HCC development and progression is needed.

There is considerable interest in the possibility of pharmaceutical inhibition of HSD17B13 to counteract the steatogenic effect of over expressed HSD17B13, and mimic the loss-of-function derived from the HSD17B13 rs72613567:TA variant. For example, fenofibrate which is an agonist of the peroxisome proliferator-activated receptor- $\alpha$ (PPAR $\alpha$ ) and approved for use in humans, suppresses hepatic HSD17B13 expression in mouse liver, ${ }^{50}$ a finding supported by the high expression of HSD17B13 observed in ppar $\alpha$ knock-out mice. ${ }^{42}$

This study has a number of strengths. The study cohorts were large and well-characterized and specifically selected to explore the genetic risks associated with the development of alcoholrelated cirrhosis and HCC in Caucasians of European origin. Controls were exercised for a number of known risk factors such as age, sex, BMI and diabetes. Similar effect sizes were observed across all individual geographical cohorts excluding the possibility of heterogeneity and, thus, broadening the applicability of the findings. The specificity of the genetic associations was explored by including a large control cohort of alcohol misusers who had no evidence of liver disease.

This study also has some limitations; first, it was cross-sectional and undertaken retrospectively, meaning that phenotypic data sets were not always complete. Second, it is likely that some of the patients with alcohol-related cirrhosis will develop HCC over time and equally likely that some of the individuals misusing alcohol will develop cirrhosis in the future, particularly if they continue to drink. However, the group differences were significant and remained robust to adjustment for confounders, including age. Finally, the findings can not be used to define the genetic risks associated with the development of cirrhosis and HCC in people with liver disease of different aetiologies or from outside of Europe, as pointed out in relation to the findings of Chen and colleagues. ${ }^{49}$

In conclusion: HSD17B13 rs72613567:TA protects against the development of alcohol-related cirrhosis and, at least in men, the subsequent development of HCC. Its carriage attenuates the increased risk associated with carriage of PNPLA3 rs738409:G. Combining phenotypic and genetic signatures to score risk could facilitate management of patients with alcohol-related liver 
disease. Further evaluation of the function of HSD17B13 and the rs72613567 variant may identify suitable drug targets.

\section{Acknowledgements}

The authors thank the Clinical Research Support Service of the CHUV-UNIL, Lausanne, Switzerland for providing the infrastructure for patient recruitment and collecting phenotypic data.

This article is protected by copyright. All rights reserved 


\section{REFERENCES (Author names in bold designate shared co-first authorship)}

1. Singal AK, Bataller R, Ahn J, Ahn J, Kamath PS, Shah VH. ACG Clinical Guideline: Alcoholic Liver Disease. Am J Gastroenterology 2018;113:175-194.

2. Asrani SK, Devarbhavi H, Eaton J, Kamath PS. Burden of liver diseases in the world. J Hepatol 2019;70:151-171.

3. Rehm J, Samokhvalov AV, Shield KD. Global burden of alcoholic liver diseases. J Hepatol 2013;59:160-168.

4. Burra P, Senzolo M, Adam R, Delvart V, Karam V, Germani G, et al. Liver transplantation for alcoholic liver disease in Europe: a study from the ELTR (European Liver Transplant Registry). Am J Transplant 2010;10:138-148.

5. Lelbach WK. Leberschaden bei chronischem Alcoholismuss I-III. Acta Hepatosplenologica, 1966; 13: 321-349.

6. Leevy CM. Cirrhosis in alcoholics. Medical Clinics of North America, 1968; 52: 1445-1451.

7. Teli MR, Day CP, Burt AD, Bennett MK, James OF. Determinants of progression to cirrhosis or fibrosis in pure alcoholic fatty liver. Lancet 1995;346:987-990.

8. Kodama K, Tokushige K, Hashimoto E, Taniai M, Shiratori K. Hepatic and extrahepatic malignancies in cirrhosis caused by nonalcoholic steatohepatitis and alcoholic liver disease. Alcohol Clin Exp Res 2013;37:E247-252.

9. Mancebo A, Gonzalez-Dieguez ML, Cadahia V, Varela M, Pérez R, Navascués CA, et al. Annual incidence of hepatocellular carcinoma among patients with alcoholic cirrhosis and identification of risk groups. Clin Gastroenterol Hepatol 2013;11:95-101.

10. Ganne-Carrié N, Chaffaut C, Bourcier V, Archambeaud I, Perarnau JM, Oberti F, et al. Estimate of hepatocellular carcinoma incidence in patients with alcoholic cirrhosis. J Hepatol 2018;69:1274-1283.

11. Ganne-Carrié N, Nahon P. Hepatocellular carcinoma in the setting of alcohol-related liver disease. J Hepatol 2019;70:284-293.

12. Kim D, Li AA, Perumpail BJ, Gadiparthi C, Kim W, Cholankeril G, et al. Changing trends in etiology- and ethnicity-based annual mortality rates of cirrhosis and hepatocellular carcinoma in the United States. Hepatology 2019;69:1064-1074. 
13. Stickel F, Moreno C, Hampe J, Morgan MY. Genetics of alcohol dependence and alcoholrelated liver disease. J Hepatol 2017;66:195-211.

14. Stickel F, Buch S, Lau K, Meyer zu Schwabedissen H, Berg T, Ridinger M, et al. Genetic variation in the PNPLA3 gene is associated with alcoholic liver injury in Caucasians. Hepatology 2011;53:86-95.

15. Chamorro AJ, Torres JL, Mirón-Canelo JA, González-Sarmiento R, Laso FJ, Marcos M. Systematic review with meta-analysis: the I148M variant of patatin-like phospholipase domain-containing 3 gene (PNPLA3) is significantly associated with alcoholic liver cirrhosis. Aliment Pharmacol Ther 2014;40:571-581.

16. Singal AG, Manjunath $H$, Yopp AC, Beg MS, Marrero JA, Gopal P, et al. The effect of PNPLA3 on fibrosis progression and development of hepatocellular carcinoma: a meta-analysis. Am J Gastroenterol 2014;109:325-34.

17. Trépo E, Nahon P, Bontempi G, Valenti L, Falleti E, Nischalke HD, et al. Association between the PNPLA3 (rs738409 C>G) variant and hepatocellular carcinoma: Evidence from a metaanalysis of individual participant data. Hepatology 2014;59:2170-7.

18. Stickel F, Buch S, Nischalke HD, Weiss KH, Gotthardt D, Fischer J, et al. Genetic variants in PNPLA3 and TM6SF2 predispose to the development of hepatocellular carcinoma in individuals with alcohol-related cirrhosis. Am J Gastroenterol 2018;113:1475-1483.

19. Buch S, Stickel F, Trépo E, Way M, Herrmann A, Nischalke HD, et al. A two-stage genomewide association study confirms PNPLA3 and identifies TM6SF2 and MBOAT7 as novel risk loci for alcohol-related cirrhosis. Nat Genet 2015;47:1443-1448.

20. Abul-Husn NS, Cheng $X$, Li AH, Xin Y, Schurmann $C$, Stevis $P$, et al. A protein-truncating HSD17B13 Variant and protection from chronic liver disease. N Engl J Med 2018;378:10961106.

21. Yang J, Trépo E, Nahon P, Cao Q, Moreno C, Letouzé E, et al. A HSD17B13 variant protects from hepatocellular carcinoma development in alcoholic liver disease. Hepatology 2019;70:231-240.

22. EASL. Clinical Practice Guidelines: Management of hepatocellular carcinoma. J Hepatol 2018;69:182-236.

This article is protected by copyright. All rights reserved 
23. Pugh RN, Murray-Lyon IM, Dawson JL, Pietroni MC, Williams R. Transection of the oesophagus for bleeding oesophageal varices. Br J Surg 1973;60:646-9.

24. American Psychiatric Association. Diagnostic and statistical manual of mental disorders 4th ed. Washington DC: American Psychiatric Press; 1994.

25. Witte JS, Visscher PM, Wray NR. The contribution of genetic variants to disease depends on the ruler. Nat Rev Genet 2014;15:765-76.

26. Anderson CA, Pettersson FH, Clarke GM, Cardon LR, Morris AP, Zondervan KT. Data quality control in genetic case-control association studies. Nat Protoc 2010;5:1564-73.

27. 1000 Genomes Project Consortium, Auton A, Brooks LD, Durbin RM, Garrison EP, Kang HM, Korbel JO, et al. A global reference for human genetic variation. Nature 2015;526:68-74.

28. Li D, Zhao H, Gelernter J. Strong association of the alcohol dehydrogenase 1B gene (ADH1B) with alcohol dependence and alcohol-induced medical diseases. Biol Psychiatry 2011;70:504-512.

29. Li D, Zhao H, Gelernter J. Strong protective effect of the aldehyde dehydrogenase gene $(A L D H 2)$ 504lys $\left({ }^{*} 2\right)$ allele against alcoholism and alcohol-induced medical diseases in Asians. Hum Genet 2012;131:725-737.

30. Lee Fl. Cirrhosis and hepatoma in alcoholics. Gut 1966;7: 77-85.

31. Schlichting P, Christensen E, Andersen PK, Fauerholdt L, Juhl E, Poulsen H, et al. Prognostic factors in cirrhosis identified by Cox's regression model. Hepatology 1983;3:889-95.

32. D'Amico G, Morabito A, Pagliaro L, Marubini E. Survival and prognostic indicators in compensated and decompensated cirrhosis. Dig Dis Sci 1986;31:468-75.

33. Ginés $\mathrm{P}$, Quintero $\mathrm{E}$, Arroyo $\mathrm{V}$, Terés J, Bruguera $\mathrm{M}$, Rimola A, et al. Compensated cirrhosis: natural history and prognostic factors. Hepatology 1987;7:122-8.

34. Meffert PJ, Repp KD, Völzke H, Weiss FU, Homuth G, Kühn JP, et al. The PNPLA3 SNP rs738409:G allele is associated with increased liver disease-associated mortality but reduced overall mortality in a population-based cohort. J Hepatol 2018;68:858-60.

35. Atkinson SR, Way MJ, McQuillin A, Morgan MY, Thursz MR. Reply to: The PNPLA3 SNP rs738409:G allele is associated with increased liver disease-associated mortality but reduced overall mortality in a population-based cohort. J Hepatol 2018;68:860-2. 
36. Ferenci P, Pfeiffenberger J, Stättermayer A, Stauber RE, Willheim C, Weiss KH, et al. A HSD17B13 variant as genetic modifier in hepatic Wilson Disease. JHep Rep 2019; doi.org/10.1016/j.jhepr.2019.02.007.

37. Saloniemi T, Jokela H, Strauss L, Pakarinen P, Poutanen M. The diversity of sex steroid action: novel functions of hydroxysteroid $(17 \beta)$ dehydrogenases as revealed by genetically modified mouse models. J Endocrinol 2012;212:27-40.

38. Su W, Peng J, Li S, Dai YB, Wang CJ, $\mathrm{Xu} \mathrm{H}$, et al. Liver $X$ receptor $\alpha$ induces $17 \beta$ hydroxysteroid dehydrogenase-13 expression through SREBP-1c. Am J Physiol Endocrinol Metab 2017;312:E357-E367.

39. Su W, Wang Y, Jia X, Wu W, Li L, Tian X, et al. Comparative proteomic study reveals $17 \beta$ HSD13 as a pathogenic protein in nonalcoholic fatty liver disease. Proc Natl Acad Sci U S A 2014;111:11437-42.

40. Kampf C, Mardinoglu A, Fagerberg L, Hallström BM, Edlund K, Lundberg E, et al. The human liver-specific proteome defined by transcriptomics and antibody-based profiling. FASEB J 2014;28:2901-14.

41. Adam M, Heikelä H, Sobolewski C, Portius D, Mäki-Jouppila J, Mehmood A, et al. Hydroxysteroid (17ß) dehydrogenase 13 deficiency triggers hepatic steatosis and inflammation in mice. FASEB J 2018;32:3434-3447.

42. Horiguchi Y, Araki M, Motojima K. 17beta-Hydroxysteroid dehydrogenase type 13 is a liverspecific lipid droplet-associated protein. Biochem Biophys Res Commun 2008;370:235-8.

43. Ma Y, Belyaeva OV, Brown PM, Fujita K, Valles K, Karki S, et al. HSD17B13 is a hepatic retinol dehydrogenase associated with histological features of non-alcoholic fatty liver disease. Hepatology 2019;69:1504-1519.

44. Saeed A, Dullaart RPF, Schreuder TCMA, Blokzijl H, Faber KN. Disturbed vitamin A metabolism in non-alcoholic fatty liver disease (NAFLD). Nutrients 2017;10, pii: E29. doi.org/10.3390/nu10010029.

45. Kim SC, Kim CK, Axe D, Cook A, Lee M, Li T, et al. All-trans-retinoic acid ameliorates hepatic steatosis in mice by a novel transcriptional cascade. Hepatology 2014;59:1750-60.

46. Wang XD, Liu C, Chung J. Chronic alcohol intake reduces retinoic acid concentration and enhances AP-1 (c-jun and c-fos) expression in rat liver. Hepatology 1998;28:744-750. 
47. Cortes E, Lachowski D, Rice A, Chronopoulos A, Robinson B, Thorpe S, et al. Retinoic acid receptor- $\beta$ is downregulated in hepatocellular carcinoma and cirrhosis and its expression inhibits myosin-driven activation and durotaxis in hepatic stellate cells. Hepatology 2019;69:785-802.

48. Muto $Y$, Moriwaki H, Saito A. Prevention of second primary tumors by an acyclic retinoid in patients with hepatocellular carcinoma. N Engl J Med 1999;340:1046-7.

49. Chen J, Zhuo JY, Yang F, Liu ZK, Zhou L, Xie HY, et al. 17-beta-hydroxysteroid dehydrogenase 13 inhibits the progression and recurrence of hepatocellular carcinoma. Hepatobiliary Pancreat Dis Int 2018;17:220-226.

50. Rotroff DM, Pijut SS, Marvel SW, Jack JR, Havener TM, Pujol A, et al. Genetic variants in HSD17B3, SMAD3, and IPO11 impact circulating lipids in response to fenofibrate in individuals with type 2 diabetes. Clin Pharmacol Ther 2018;103:712-721.

This article is protected by copyright. All rights reserved 


\section{Legends to figures}

\section{Figure 1}

Forrest plot showing genotypic ORs for heterozygous and homozygous carriage of the HSD17B13 rs72613567:TA allele. Figure 1A: the unadjusted analysis; Figure 1B analysis adjusted for age, sex, body mass index (BMI), type 2 diabetes mellitus (T2DM) and country. Homozygous carriage of HSD17B13 rs72613567:T wild type is set as a reference.

\section{Figure 2}

A: Relative proportions of patients with alcohol-related cirrhosis (CIRR) according to gene signature comprised of HSD17B13 rs72613567 and PNPLA3 rs739409 genotypes in relation to alcohol misusers without liver disease (ALC).

B: Relative proportions of patients with HCC according to gene signature comprised of HSD17B13 rs72613567 and PNPLA3 rs739409 genotypes in relation to patients with alcohol-related cirrhosis (CIRR).

C: Proportions of HCC patients according to gene signature comprised of HSD17B13 rs72613567 and PNPLA3 rs739409 genotypes in relation to alcohol misusers with no liver disease (ALC).

\section{Figure 3}

A: Test for trend on the proportion of HSD17B13 rs72613567 genotypes in the three cohorts showing a gradual decrease in TA allele carriage with escalating severity of liver disease.

B: Test for trend on the proportion of HSD17B13 rs72613567 genotypes in women showing a decrease in TA carriage in patients with cirrhosis but not in patients with HCC.

C: Test for trend on the proportion of HSD17B13 rs72613567 genotypes in men showing a gradual decrease of TA allele carriage with escalating severity of liver disease.

This article is protected by copyright. All rights reserved 
Table 1: Demographic characteristics of the study cohorts

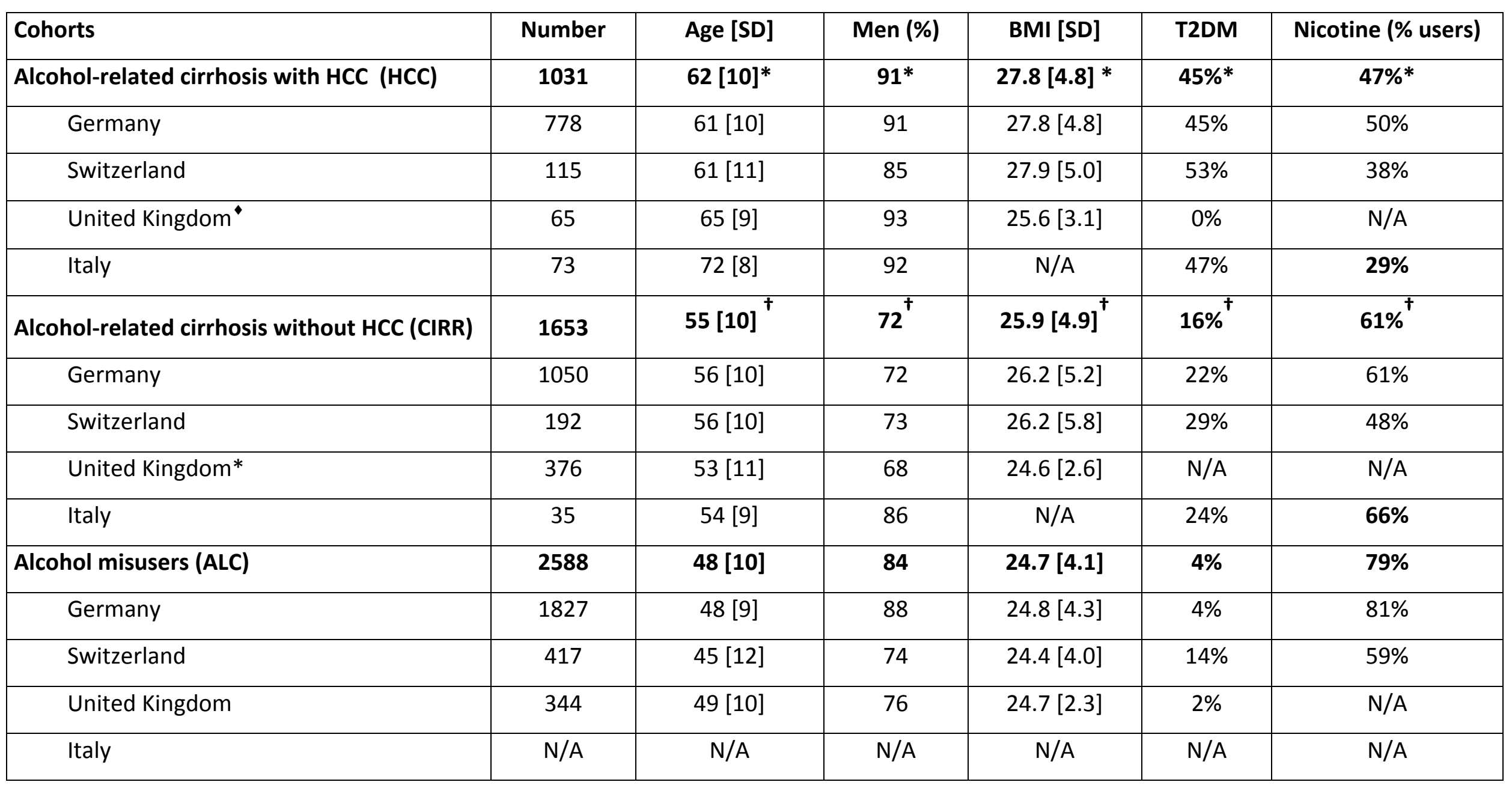

BMI, body mass index; T2DM, type II diabetes mellitus; N/A, not available;

- People with a BMI> 30 and/or with type II diabetes were excluded a priori;

This article is protected by copyright. All rights reserved 
Significance of the difference between patients with alcohol-related cirrhosis with and without HCC: ${ }^{*} p<0.001$;

Significance of the difference between patients with alcohol-related cirrhosis and alcohol misusers: ${ }^{\dagger} p<0.001$

This article is protected by copyright. All rights reserved 
Table 2: Univariate and multivariate analyses for association of HSD17B13 rs72613567 with alcohol-related cirrhosis and HCC in the total European cohort

\begin{tabular}{|c|c|c|c|c|c|c|c|c|c|}
\hline \multirow[t]{2}{*}{ Cohorts } & \multirow[t]{2}{*}{$\begin{array}{c}\text { HSD17B13 } \\
\text { (rs72613567) }\end{array}$} & \multicolumn{2}{|c|}{$\begin{array}{c}\text { Comparative } \\
\text { groups }\end{array}$} & \multirow{2}{*}{$\begin{array}{c}\text { Genotypic } \\
\text { OR }(95 \% \mathrm{Cl}) \\
\text { regression model: A }\end{array}$} & \multirow{2}{*}{$\begin{array}{c}\text { Genotypic } \\
\text { OR }(95 \% \mathrm{Cl}) \\
\text { regression model: B }\end{array}$} & \multirow[t]{2}{*}{$\begin{array}{c}\text { Regression } \\
\text { model }\end{array}$} & \multirow[t]{2}{*}{$\begin{array}{l}\text { Allelic OR } \\
\text { (95\% Cl) }\end{array}$} & \multirow[t]{2}{*}{$\begin{array}{c}\text { Significance } \\
\text { (P) }\end{array}$} & \multirow{2}{*}{$\begin{array}{c}\text { Cases/ } \\
\text { controls } \\
\text { (n) }\end{array}$} \\
\hline & & HCC & CIRR & & & & & & \\
\hline \multirow{4}{*}{$\begin{array}{l}\text { Alcoholic- } \\
\text { related cirrhosis } \\
\text { and HCC } \\
\text { (HCC) }\end{array}$} & $\mathrm{T} \mid \mathrm{T}$ & 705 & 1027 & & & & & & \\
\hline & T|TA & 287 & 524 & $0.80(0.67-0.95)$ & $0.92(0.69-1.22)$ & $A$ & $0.77(0.68-0.89)$ & $2.27 \times 10^{-4}$ & $1031 / 1653$ \\
\hline & TA|TA & 39 & 102 & $0.56(0.38-0.82)$ & $0.43(0.23-0.80)$ & $B$ & $0.79(0.63-0.99)$ & 0.037 & $604 / 679$ \\
\hline & MAF & 0.177 & 0.220 & $P=1.05 \times 10^{-3}$ & $P=0.028$ & & & & \\
\hline & & CIRR & ALC & & & & & & \\
\hline \multirow{4}{*}{$\begin{array}{l}\text { Alcohol-related } \\
\text { cirrhosis } \\
\text { without HCC } \\
\text { (CIRR) }\end{array}$} & $\mathrm{T} \mid \mathrm{T}$ & 1027 & 1401 & & & & & & \\
\hline & $\mathrm{T} \mid \mathrm{TA}$ & 524 & 1009 & $0.71(0.62-0.81)$ & $0.79(0.63-0.98)$ & $A$ & $0.79(0.72-0.88)$ & $8.13 \times 10^{-6}$ & $1653 / 2588$ \\
\hline & TA|TA & 102 & 178 & $0.78(0.61-1.01)$ & $0.82(0.54-1.23)$ & B & $0.85(0.72-0.99)$ & 0.048 & $679 / 1483$ \\
\hline & MAF & 0.220 & 0.264 & $P=1.54 \times 10^{-6}$ & $P=0.085$ & & & & \\
\hline & & $\mathrm{HCC}$ & ALC & & & & & & \\
\hline Alcohol & $\mathrm{T} \mid \mathrm{T}$ & 705 & 1401 & & & & & & \\
\hline
\end{tabular}

This article is protected by copyright. All rights reserved 


\begin{tabular}{|c|c|c|c|c|c|c|c|c|c|}
\hline \multirow{3}{*}{$\begin{array}{c}\text { misusers } \\
\text { (ALC) }\end{array}$} & $\mathrm{T} \mid \mathrm{TA}$ & 287 & 1009 & $0.57(0.48-0.66)$ & $0.68(0.52-0.89)$ & A & $0.60(0.53-0.69)$ & $1.69 \times 10^{-14}$ & $1031 / 2588$ \\
\hline & TA|TA & 39 & 178 & $0.44(0.30-0.62)$ & $0.39(0.21-0.72)$ & B & $0.66(0.53-0.81)$ & $1.14 \times 10^{-4}$ & $604 / 1483$ \\
\hline & MAF & 0.177 & 0.264 & $P=3.32 \times 10^{-14}$ & $P=6.00 \times 10^{-4}$ & & & & \\
\hline
\end{tabular}

$\mathrm{Cl}$, confidence intervals; MAF, minor allele frequency; OR, odds ratio

Genotypic and allelic odds ratios were assessed by logistic regression models: model A - univariate logistic regression; model B -multivariate logistic regression adjusted for age, sex, body mass index, type 2 diabetes mellitus and country.

This article is protected by copyright. All rights reserved 
Table 3: Univariate and multivariate analyses for association of PNPLA3 rs738409 with alcohol-related cirrhosis and HCC in the total European cohort

\begin{tabular}{|c|c|c|c|c|c|c|c|c|c|}
\hline Cohorts & $\begin{array}{c}\text { PNPLA3 } \\
\text { (rs738409) }\end{array}$ & $\begin{array}{r}\text { Comp } \\
\text { grc }\end{array}$ & $\begin{array}{l}\text { rative } \\
\text { ups }\end{array}$ & $\begin{array}{c}\text { Genotypic } \\
\text { OR }(95 \% \mathrm{Cl}) \\
\text { regression model: A }\end{array}$ & $\begin{array}{c}\text { Genotypic } \\
\text { OR }(95 \% \mathrm{Cl}) \\
\text { regression model: B }\end{array}$ & $\begin{array}{c}\text { Regression } \\
\text { model }\end{array}$ & $\begin{array}{l}\text { Allelic OR } \\
\text { (95\% CI) }\end{array}$ & $\begin{array}{c}\text { Significance } \\
\text { (P) }\end{array}$ & $\begin{array}{c}\text { Cases/ } \\
\text { controls } \\
\text { (n) }\end{array}$ \\
\hline \multirow{5}{*}{$\begin{array}{c}\text { Alcohol-related } \\
\text { cirrhosis with HCC } \\
\text { (HCC) }\end{array}$} & & $\mathrm{HCC}$ & CIRR & & & & & & \\
\hline & $\mathrm{C} \mid \mathrm{C}$ & 269 & 697 & & & & & & \\
\hline & $C \mid G$ & 497 & 714 & $1.80(1.50-2.16)$ & $1.75(1.30-2.36)$ & A & $1.77(1.58-1.98)$ & $2.31 \times 10^{-23}$ & $1030 / 1631$ \\
\hline & $\mathrm{G} \mid \mathrm{G}$ & 264 & 220 & $3.11(2.48-3.90)$ & $2.75(1.88-4.01)$ & B & $1.67(1.39-2.01)$ & $7.19 \times 10^{-8}$ & $603 / 660$ \\
\hline & MAF & 0.498 & 0.354 & $P=2.98 \times 10^{-22}$ & $P=4.37 \times 10^{-7}$ & & & & \\
\hline \multirow{5}{*}{$\begin{array}{l}\text { Alcohol-related } \\
\text { cirrhosis without }\end{array}$} & & CIRR & ALC & & & & & & \\
\hline & $\mathrm{C} \mid \mathrm{C}$ & 697 & 1364 & & & & & & \\
\hline & $C \mid G$ & 714 & 802 & $1.74(1.52-2.00)$ & $1.64(1.31-2.06)$ & $A$ & $1.70(1.54-1.88)$ & $1.52 \times 10^{-26}$ & $1631 / 2319$ \\
\hline & $\mathrm{G} \mid \mathrm{G}$ & 220 & 153 & $2.81(2.25-3.53)$ & $2.65(1.85-3.78)$ & B & $1.63(1.39-1.91)$ & $1.67 \times 10^{-9}$ & $660 / 1469$ \\
\hline & MAF & 0.354 & 0.239 & $P=1.61 \times 10^{-25}$ & $P=1.31 \times 10^{-8}$ & & & & \\
\hline \multirow{2}{*}{$\begin{array}{l}\text { Alcohol misuse } \\
\text { (ALC) }\end{array}$} & & HCC & ALC & & & & & & \\
\hline & $\mathrm{C} \mid \mathrm{C}$ & 269 & 1364 & & & & & & \\
\hline
\end{tabular}




\begin{tabular}{|c|c|c|c|c|c|c|l|l|l|}
\hline & $\mathrm{C} \mid \mathrm{G}$ & 497 & 802 & $3.14(2.65-3.73)$ & $2.75(2.06-3.66)$ & $\mathrm{A}$ & $3.00(2.67-3.36)$ & $2.17 \times 10^{-79}$ & $1030 / 2319$ \\
\cline { 2 - 9 } & $\mathrm{G} \mid \mathrm{G}$ & 264 & 153 & $8.75(6.90-11.10)$ & $6.76(4.60-9.95)$ & $\mathrm{B}$ & $2.63(2.18-3.17)$ & $3.89 \times 10^{-24}$ & $603 / 1469$ \\
\cline { 2 - 9 } & $\mathrm{MAF}$ & 0.498 & 0.239 & $\mathrm{P}=4.14 \times 10^{-78}$ & $\mathrm{P}=5.50 \times 10^{-23}$ & & & & \\
\hline
\end{tabular}

$\mathrm{Cl}$, confidence intervals; $\mathrm{MAF}$, minor allele frequency; OR, odds ratio

Genotypic and allelic odds ratios were assessed by logistic regression models: model A - univariate logistic regression; model B -multivariate logistic regression adjusted for age, sex, body mass index, type 2 diabetes mellitus and country

This article is protected by copyright. All rights reserved 


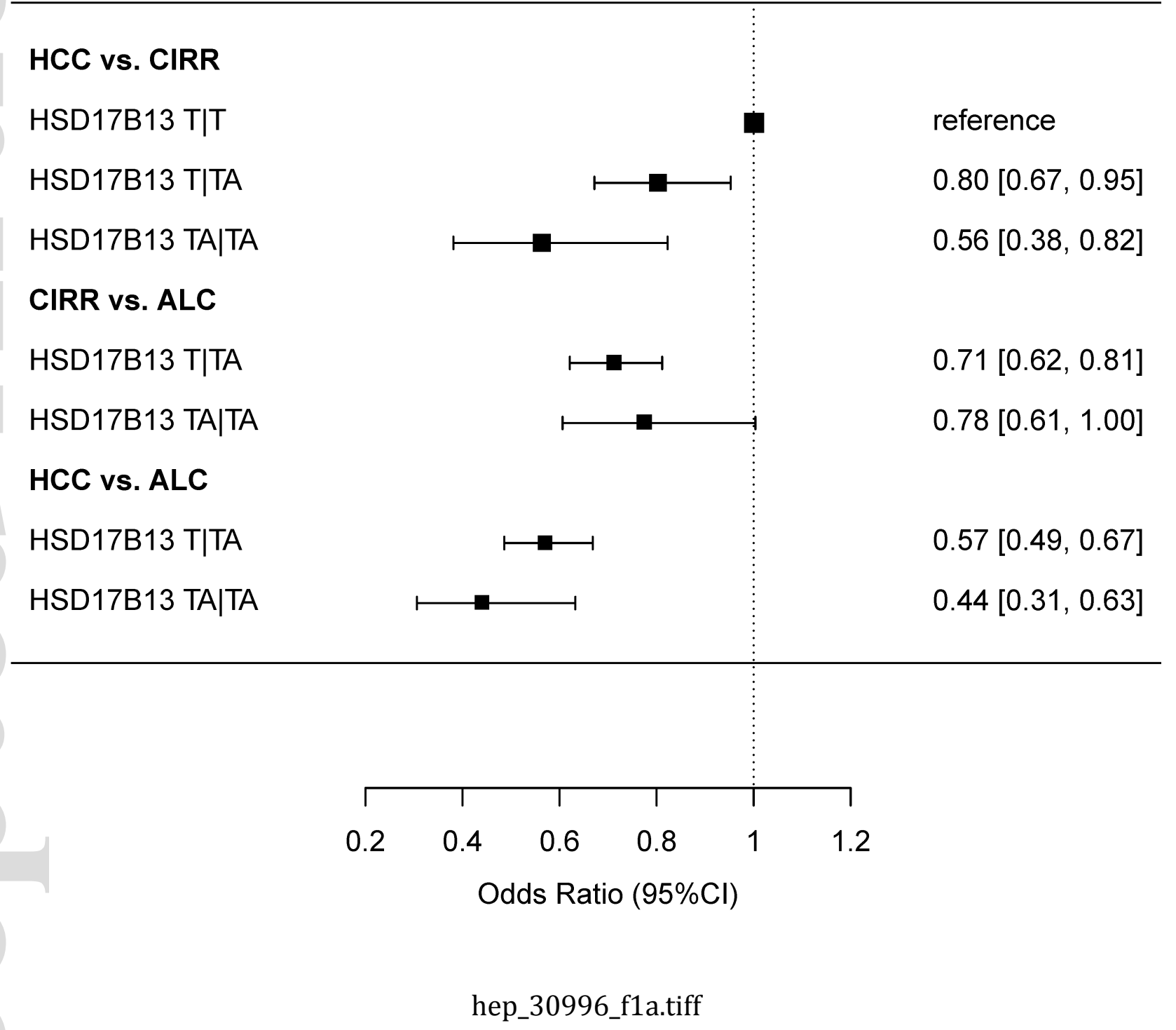




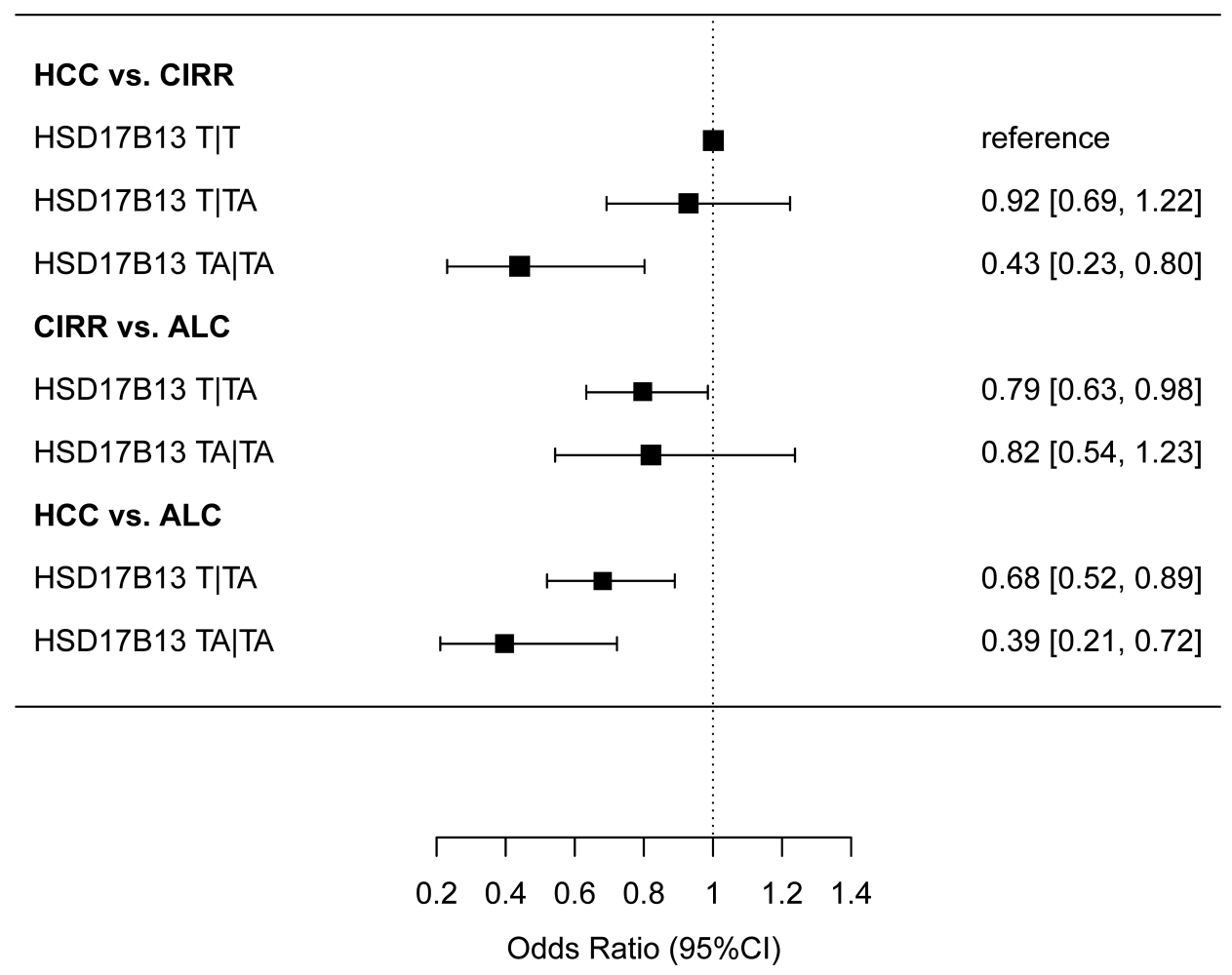

hep_30996_f1b.tif

This article is protected by copyright. All rights reserved 

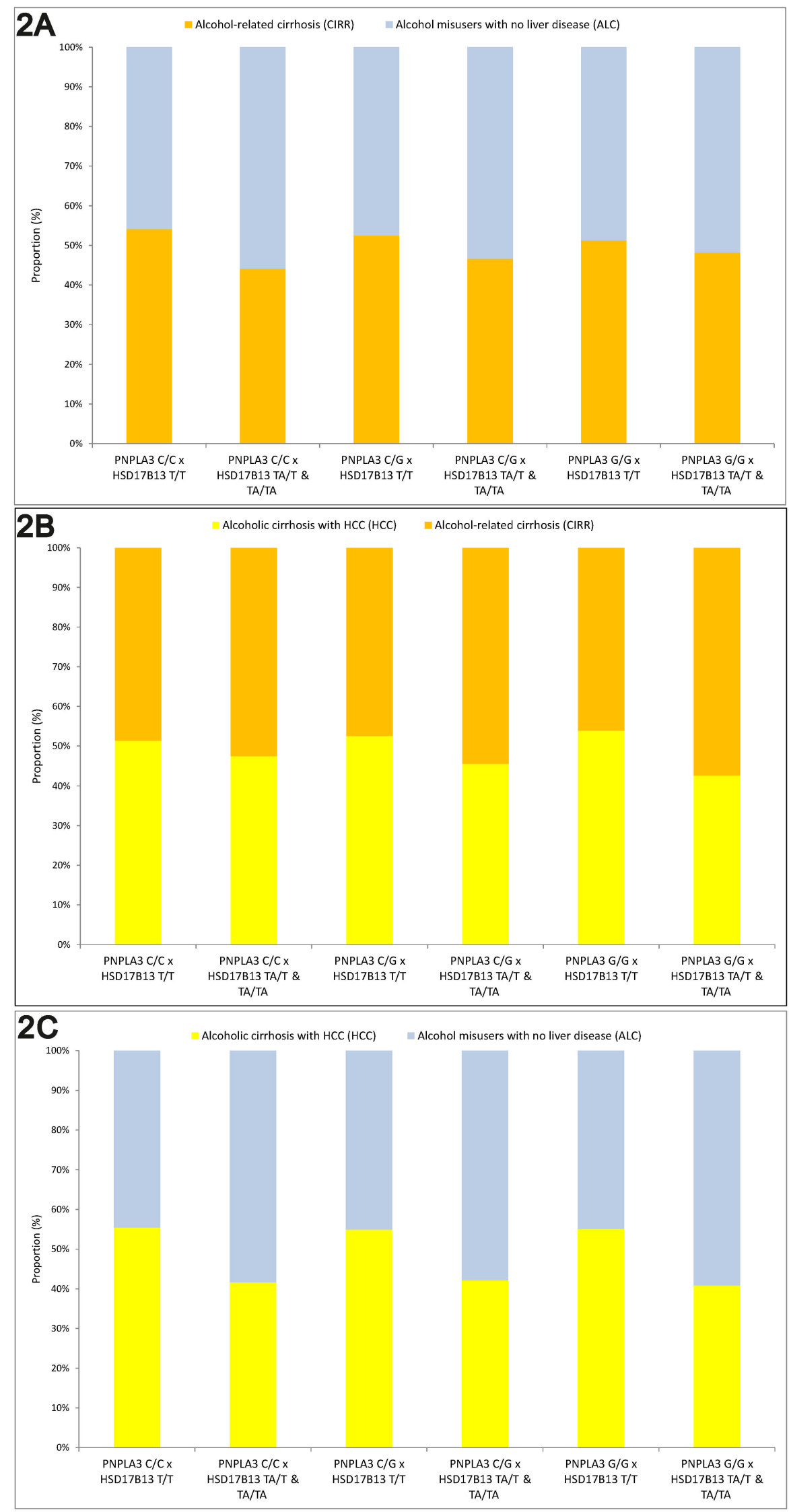

hep_30996_f2.tif 
3A Mantel-Haenszel test of trend $p=1.09 \mathrm{e}-15$

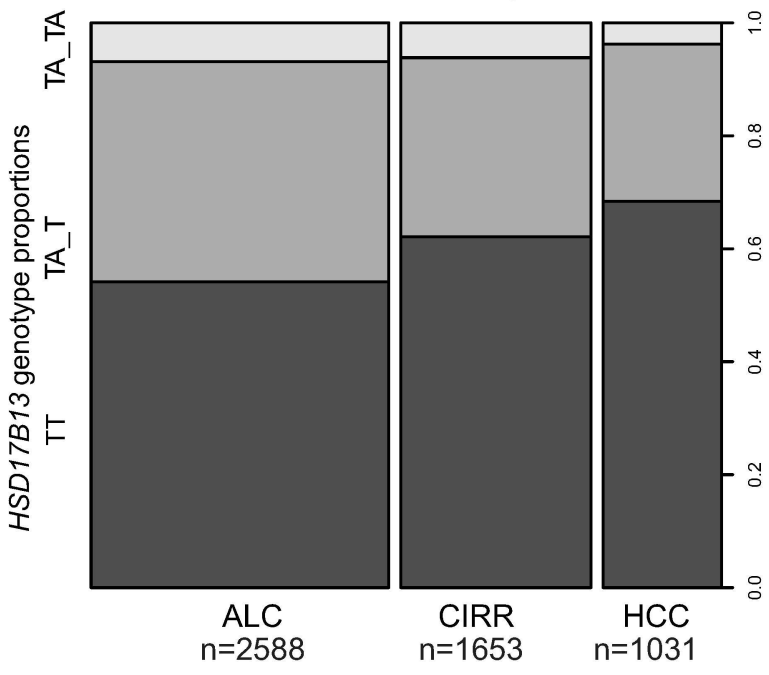

3B Mantel-Haenszel test of trend $p=0.011$

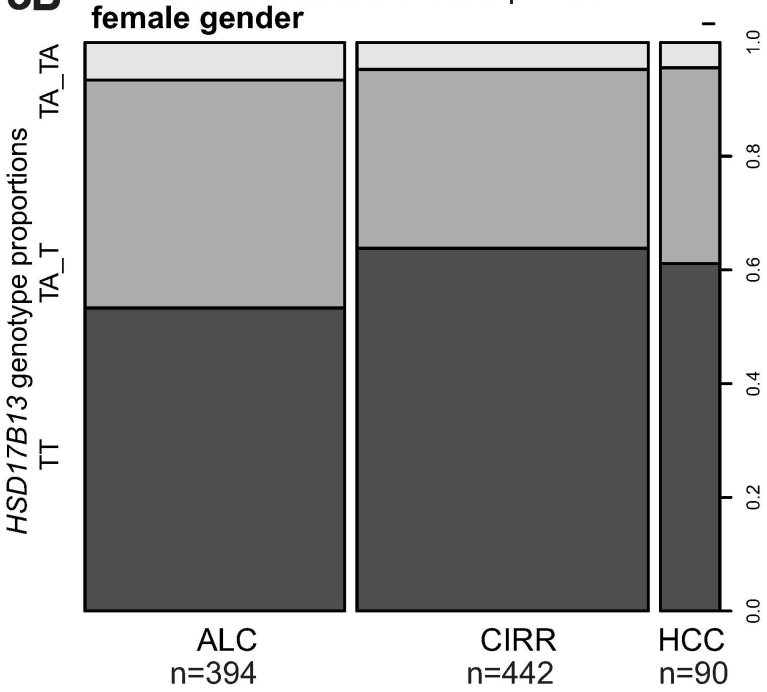

3C Mantel-Haenszel test of trend $p=1.61 \mathrm{e}-13$ male gender

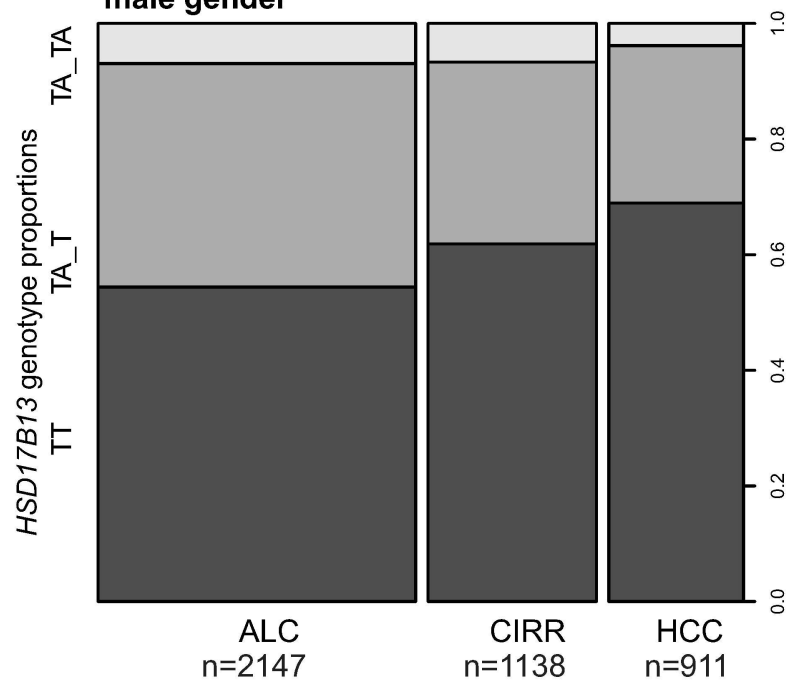

hep_30996_f3.tif 